\title{
Integrated Flight Performance Analysis of a Launch Abort System Concept
}

\author{
Paul V. Tartabini \\ NASA Langley Research Center, Hampton, VA, 23681
}

\begin{abstract}
This paper describes initial flight performance analyses conducted early in the Orion Project to support concept feasibility studies for the Crew Exploration Vehicle's Launch Abort System (LAS). Key performance requirements that significantly affect abort capability are presented. These requirements have implications on sizing the Abort Motor, tailoring its thrust profile to meet escape requirements for both launch pad and high drag/high dynamic pressure ascent aborts. Additional performance considerations are provided for the Attitude Control Motor, a key element of the Orion LAS design that eliminates the need for ballast and provides performance robustness over a passive control approach. Finally, performance of the LAS jettison function is discussed, along with implications on Jettison Motor sizing and the timing of the jettison event during a nominal mission. These studies provide an initial understanding of LAS performance that will continue to evolve as the Orion design is matured.
\end{abstract}

\section{Nomenclature}

$\alpha \quad=\quad$ Angle of attack, deg

$\sigma \quad=$ Standard deviation

$\Delta \mathrm{C}_{\mathrm{D}} \quad=\quad$ Change in drag coefficient

$\Delta$ Payload $=$ Change in payload, $1 b_{\mathrm{m}}$

$\mathrm{AM} \quad=$ Abort Motor

$\mathrm{ACM}=$ Attitude Control Motor

$\mathrm{CEV}=$ Crew Exploration Vehicle

$\mathrm{CLV}=$ Crew Launch Vehicle

$\mathrm{CM} \quad=$ Crew Module

$\mathrm{JM} \quad=$ Jettison Motor

LAS $=$ Launch Abort System

LAV $=$ Launch Abort Vehicle

PMBT $=$ Propellant Mean Bulk Temperature

RCS $=$ Reaction Control System

$\mathrm{SM}=$ Service Module

SRB $=$ Solid Rocket Booster

\section{Introduction}

In November 2005, NASA's Exploration Architecture Study (ESAS) final report was released, providing detailed analyses and initial approaches to implementing the nation's Vision for Space Exploration. ${ }^{1}$ A key element of the recommended architecture is a Crew Exploration Vehicle (CEV), similar to the Apollo/Saturn configuration, consisting of a Crew Module (CM), a Service Module (SM) and a Launch Abort System (LAS) that are mounted through a spacecraft adapter to the top of a two-stage, inline, shuttle-derived Crew Launch Vehicle (CLV). The major elements of the CEV, now called Orion, are shown in Fig. 1.

While many features of the architecture addressed important program objectives such as affordability and rapid development, perhaps the most important was the need for a robust abort capability that provides an order-of-

\footnotetext{
• Aerospace Engineer, Vehicle Analysis Branch, Mail Stop 451, Member AIAA.
} 
magnitude improvement in safety over the Space Shuttle. To achieve this objective, Orion is being designed with the capability to abort at any time throughout its mission.

There are a number of challenges associated with providing continuous abort coverage during ascent. The CLV, now called Ares I, consists of a single Shuttlederived five-segment solid rocket booster (SRB) first stage and an upper stage powered by a liquid oxygen/liquid hydrogen J-2X engine. For Orion, the LAS and SM provide continuous abort coverage across the

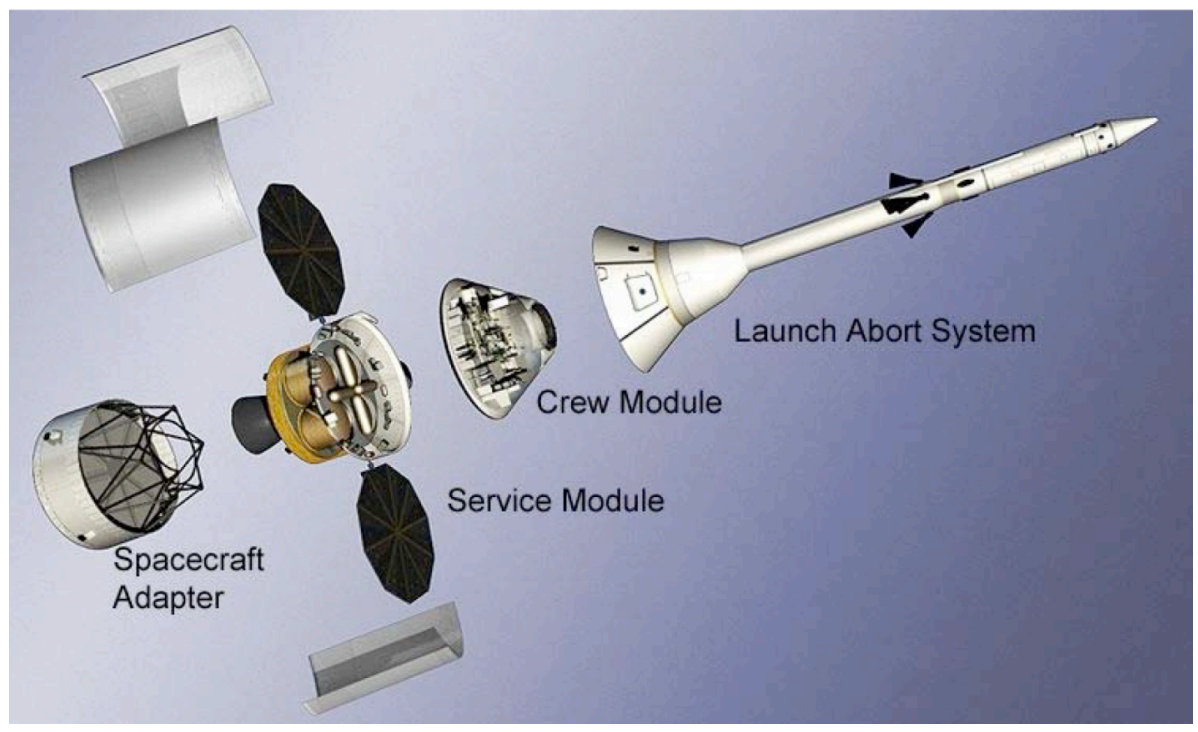

Figure 1. Elements of the Orion Crew Exploration Vehicle.

entire launch envelope. ${ }^{2}$ Similar to Apollo, a dedicated LAS is required to perform aborts off the launch pad as well as during the high acceleration and high dynamic pressure phases of flight seen during SRB operation. Also like Apollo, the Orion LAS utilizes a tractor rocket tower configuration mounted to the front of the CM to pull the crew to safety. ${ }^{3}$ The LAS is the only means of abort from the pad until shortly after upper stage J-2X ignition. At this point the LAS is jettisoned to increase CLV payload performance, and ascent aborts are performed using the SM propulsion system.

The purpose of this paper is to discuss some of the initial flight performance analyses that were conducted early in the Orion Project to establish concept feasibility for the Launch Abort System. The LAS will continue to evolve over the next years as the Orion design is matured, and these analyses will be updated accordingly. The focus here was on defining and understanding the major performance requirements that drive the LAS design and investigating its abort capability across a range of bounding flight conditions. Because LAS aborts involve highly dynamic flight occurring at high acceleration levels, simulation studies were performed using a multi-body, six-degree-of-freedom abort simulation. Simulation results are presented that examine altitude and downrange requirements during pad aborts, separation distance between the aborting vehicle and CLV during ascent aborts and LAS jettison performance during nominal missions and abort scenarios.

\section{Launch Abort System}

The Orion LAS design configuration considered in this paper is shown in Fig. 2. As previously mentioned, the specific LAS configuration will continue to change as the Orion CEV design matures. The LAS is mounted to the top of the CM through the adapter cone, which is designed to carry loads during an abort. The combined LAS/CM abort configuration is referred to as the Launch Abort Vehicle (LAV).

The LAS utilizes three different solid rocket motors to carry out its basic functions. Escape capability is provided by the Abort Motor (AM) consisting of four exposed nozzles canted 25 deg off the centerline to reduce plume impingement on the $\mathrm{CM}$. The AM is a reverse flow configuration, a key difference from Apollo, in which exhaust gases are redirected through a

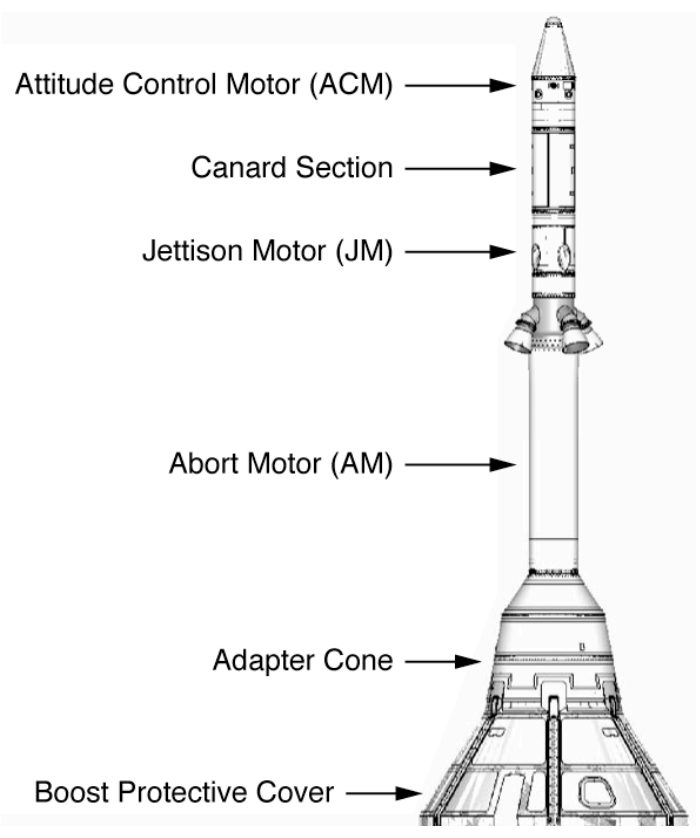

Figure 2. Orion Launch Abort System considered in this paper. 
180 deg angle, thus enabling the nozzles to be moved farther away from the $\mathrm{CM}$, to further reduce plume impingement loads and allow a smaller cant angle with fewer cosine losses. The boost protective cover (BPC) provides the $\mathrm{CM}$ with additional protection from $\mathrm{AM}$ exhaust gases.

The AM thrust profile varies over the $\sim 4$ sec burn duration, providing a peak thrust-to-weight of roughly 15 early in the burn. The shape of the thrust profile is important for meeting performance requirements. High thrust is initially required to achieve ample separation distance from the CLV during a high drag abort. At the same time, acceleration levels and their duration must not exceed crew safety or structural design limits. Also important is total impulse, which is driven by the need during a pad abort to achieve enough altitude to ensure a successful parachute deployment, and enough downrange distance to ensure landing on safe terrain, far from the thermal radiation effects of a fireball.

Another key difference from the Apollo launch escape system is with regard to control. Apollo utilized a passive system that relied on $\sim 1,000 \mathrm{lb}_{\mathrm{m}}$ of ballast to keep the abort vehicle passively stable during the escape rocket burn, and fixed canards that were deployed at a predetermined time to provide a destabilizing aerody-

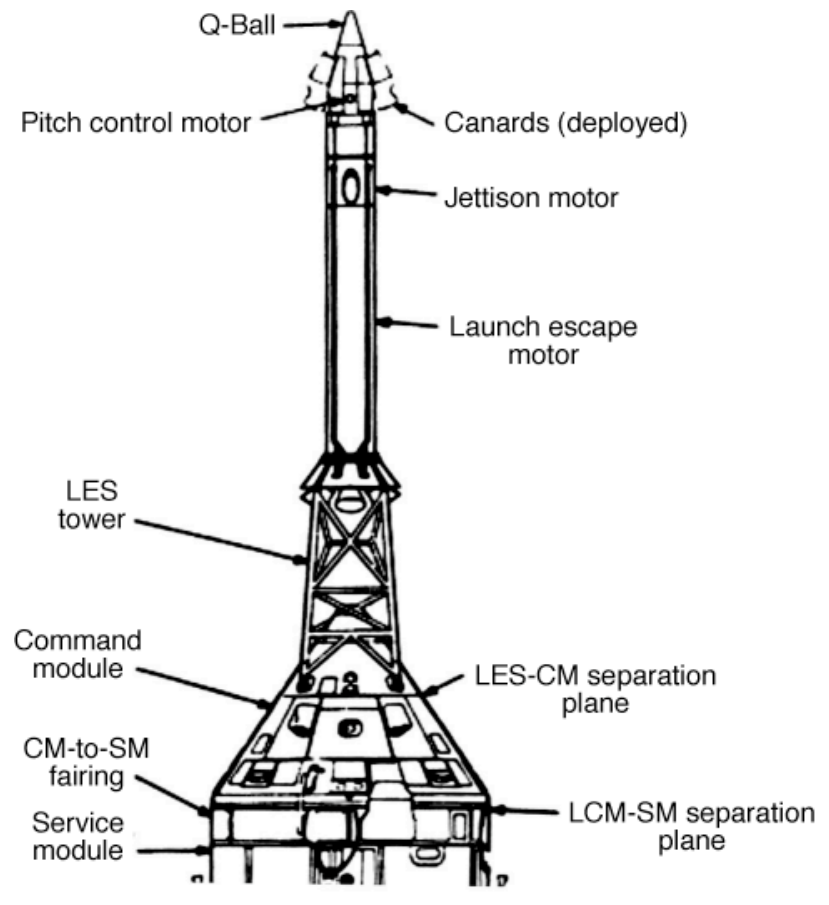

Figure 3. Apollo Launch Escape System. namic moment to reorient the vehicle to the heat shield forward attitude required for parachute deployment. The canards also damped out oscillations after reorientation, but were not effective at high altitudes where it was possible for the abort vehicle to tumble until sufficient dynamic pressure built up during descent. In addition, Apollo utilized a short-duration pitch control motor oriented 90 deg to the vehicle centerline for aborts during the first $42 \mathrm{sec}$ of nominal ascent. The pitch motor was ignited at the same time as the escape rocket and imparted an initial rotational rate to achieve lateral downrange and separation. For reference, the Apollo Launch Escape System is shown in Fig. 3.

By contrast, the LAS utilizes an Active Control Motor (ACM) to perform reorientation, maintain stability and provide trajectory steering capability. The ACM is a solid rocket pintle motor with eight equally spaced valves positioned circumferentially around the LAS. The valve plane is located axially near the nose to maximize the control torque about the vehicle center of gravity. Pintles are used to adjust the throat area in each valve, thus allowing control of flow direction so that the net thrust vector can be pointed in any direction normal to the vehicle centerline. The ACM can also be "nulled" so that each valve has the same flow rate and no net torque is produced.

An active control system saves weight over a passive system by eliminating the need for ballast and provides improved maneuvering capability, operation throughout the entire LAS regime and the ability to prevent the LAV from tumbling during high-altitude aborts.

The Orion LAS also includes passive canards (note: Fig. 2 shows the LAS in its canard stowed configuration) to assist the ACM and provide a fail-safe method to reorient should the ACM malfunction. Both the ACM and canards act to damp out oscillations after reorientation and steady the vehicle prior to parachute deployment. Finally, during an abort, the LAV also utilizes roll reaction control system (RCS) jets from the CM to prevent significant roll rates from building up prior to the reorientation maneuver.

The final LAS function to be discussed is jettison. The Jettison Motor (JM) is designed to perform two functions: 1) jettison a spent LAS from the CM during an abort; and 2) jettison a fully loaded LAS from the CLV during a nominal mission. Consequently, the JM is the only LAS motor that will be used on every Orion mission. The JM is sized by its nominal jettison function and is designed to provide more than $1.0-\mathrm{g}$ of relative acceleration from a thrusting CLV for $1.5 \mathrm{sec}$. The Jettison Motor consists of 4 scarfed nozzles, in which the exit plane of the nozzle is flush with the outer vehicle surface, configured such that the net thrust direction is offset 4 deg from the centerline to provide lateral separation.

A schematic showing the sequence of major events during an Orion abort is shown in Fig. 4. The AM, ACM and roll RCS are all engaged at abort initiation. Depending on when the abort occurs, a pitch maneuver may be performed. For pad and low altitude aborts, pitch rates as high as $20 \mathrm{deg} / \mathrm{sec}$ during the AM burn may be required to 


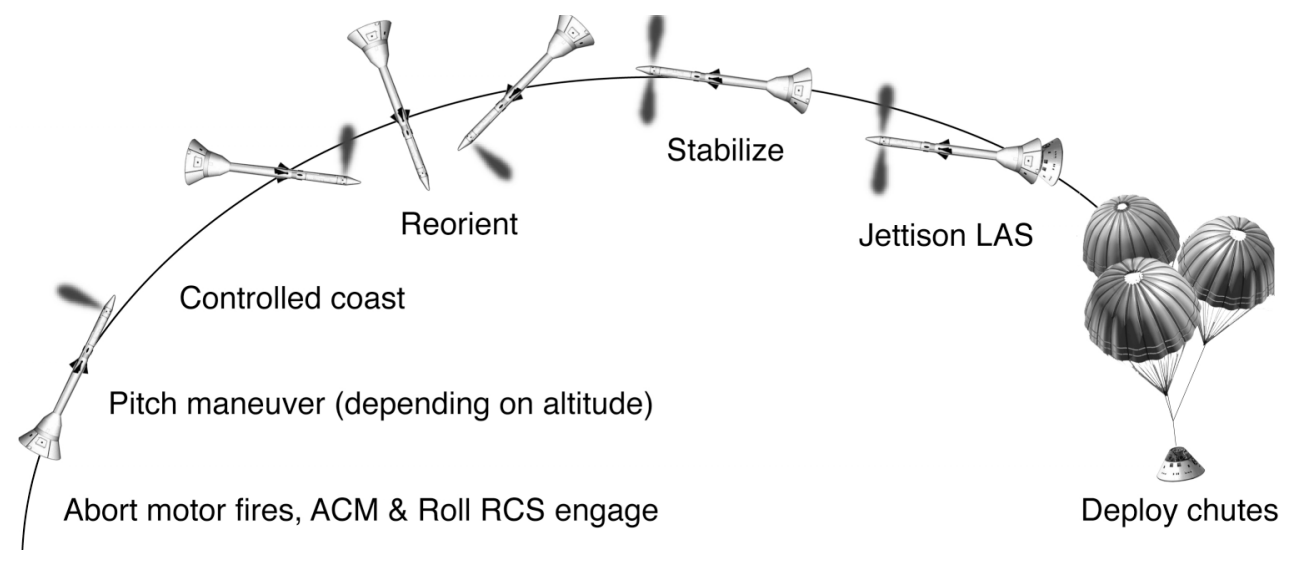

Figure 4. Sequence of key Orion abort events.

meet altitude and downrange requirements. At higher altitudes where dynamic pressure is significant, a more relaxed pitch maneuver may be performed to ensure adequate separation distance between the LAV and CLV. After the AM burns out, there is a controlled coast phase where the ACM and roll RCS act to damp out abort initiation transients as well as any disturbances induced during the AM burn. This phase is followed by a reorientation maneuver to a heat shield forward attitude. Reorientation is performed after the dynamic pressure has dropped enough to avoid excessive structural loads (typically below $120 \mathrm{psf}$ ). During high altitude aborts that occur at insignificant dynamic pressure levels, reorientation occurs sooner, typically $\sim 4 \mathrm{sec}$ after AM burnout. After reorientation the LAV is stabilized with the ACM and the spent LAS is jettisoned. For pad and low altitude aborts, the parachute deployment sequence begins immediately after jettison to maximize altitude margin. For higher altitude aborts, the CM descends to a safe parachute deployment altitude while being stabilized with its three-axis RCS system. More details on abort sequence timing can be found in Reference 4.

\section{Approach}

The analyses described in this paper were performed early in the Orion Project to assess the feasibility of the Orion LAS concept. Bounding cases were considered that included the effect of high winds and extreme Abort Motor operating conditions. The ability to abort off the launch pad, at high dynamic pressure levels and in exoatmospheric conditions was investigated, especially with regard to the major LAS functions of escape and jettison. For successful escape, sufficient altitude and downrange must be achieved during launch pad and low altitude aborts to meet parachute deploy conditions and assure safe distance from a potential launch vehicle fireball. At high dynamic pressures, sufficient separation distance from the failed launch vehicle must be rapidly achieved. For successful jettison, the LAS must be safely disposed of both during aborts after its functions have been fulfilled and also during nominal missions when its abort capability is no longer needed.

To assess LAS flight performance, a six-degree-of-freedom (6DOF) launch abort simulation was developed using the Program to Optimize Simulated Trajectories II (POST2) ${ }^{5-6}$ POST2 is a widely used, generalized simulation and trajectory optimization program first developed in the early 1970's and continuously upgraded and improved since that time. POST2 is capable of simulating multiple 3DOF or 6 DOF bodies and can be easily modified by an experienced user to include vehicle guidance, navigation and control systems and non-standard trajectory models. These features were required to simulate the multiple bodies involved in an Orion abort scenario and to include the control algorithm used to drive ACM and RCS behavior.

The POST2 studies assumed a CM abort weight of $18.9 \mathrm{klb}$ and a fully loaded LAS weight of $14 \mathrm{klb}$. Center of gravity and inertia tensor data for all major components were modeled, and the vehicle composite quantities were adjusted throughout the simulation as propellant mass was depleted and at LAS jettison.

Aerodynamic coefficients of the vehicle configurations were obtained from a complex aerodynamic database containing data for the CM, SM, LAS and LAV developed by the CEV Aerosciences Project. The database continues to be updated with higher fidelity data as the program evolves. For the version used in this study, Crew Module data was created from a combination of CFD results and wind tunnel test data; LAV data was created primarily from CFD analysis results; and LAS data (for post-abort jettison flight) as well as LAV separation increments were based on similar Apollo-derived data. 
The POST2 analyses utilized force and moment coefficients for the basic Crew Module, LAV and LAS configurations at total angle-of-attack values ranging from 0 to $180 \mathrm{deg}$ and at Mach numbers from 0.3 to 6.0. Additional coefficient increments were applied to account for AM plume effects and deployed canards. Also, LAV separation increments were applied to approximate proximity effects between the LAV and SM, which remains attached to the CLV during a LAS abort. Finally, damping derivatives were also included for the Crew Module and LAV. Future updates will be made to the aerodynamic database to incorporate wind tunnel test data and account for higher fidelity aerodynamic effects.

Motor performance data in the form of thrust and mass flow rate tables were included in the simulation for each of the three motors. Additional AM thrust and flow rate profiles that estimated the effect of temperature and burn rate uncertainty were also included in the simulation. A proportional-integral-derivative attitude and rate feedback control law was developed to determine ACM commands to stabilize the LAV and perform pitch maneuvering and reorientation. ${ }^{4}$ In addition, CM RCS roll control was performed using phase-plane control law logic. The LAV controller was developed in the MATLAB/Simulink environment, and C source code was autogenerated by MATLAB for inclusion in the POST2 simulation. ${ }^{7}$ Each of the ACM valves and CM RCS thrusters were modeled in the POST2 simulation, and their corresponding thrust and flow rate were determined by the vehicle controller.

Wind and atmospheric properties were modeled using the 1999 Global Reference Atmosphere Model (GRAM99). ${ }^{8}$ For pad abort simulations, where better estimates of low altitude wind near the launch pad were required, wind profiles derived from measurement data at Kennedy Space Center that were representative of each month were used.

\section{Analysis Results \& Discussion}

\section{A. Pad Abort Performance}

During a launch pad abort, the LAS must pull the CM away from a potentially exploding CLV such that by the time the LAS jettison point is reached, the CM is at a high enough altitude for successful parachute deployment and far enough downrange to ensure that the $\mathrm{CM}$ is kept a safe distance from the pad, preferably landing in the water. For this study a representative altitude/downrange constraint for LAS jettison of 4,000/3,500 ft was assumed. This requirement will be refined as the Crew Module's Landing and Recovery System design matures.

Pad abort performance has a number of design implications for the LAS. First, the altitude/downrange target establishes the total impulse requirement for the Abort Motor. In addition, to maximize downrange the LAV must rapidly decrease its near-vertical initial flight path angle during the relatively short AM burn by performing a high-rate pitch over. This maneuver sets the maximum thrust requirement for the ACM and must be designed with enough control margin to prevent the LAV from tumbling. During a pad abort, the ACM also steers the LAV to a heading approximately normal to the coast line to maximize downrange over the water. After AM burnout, the ACM thrust is decreased, since less control torque is needed to perform reorientation and maintain LAV stability.

To assess worst-case pad abort performance, variations in AM performance had to be considered. First, as with any solid rocket motor, the achieved thrust level of the AM depends upon the propellant mean bulk temperature (PMBT). Launch in colder conditions results in a lower PMBT and consequently, a slower burn rate and lower thrust. Because total impulse is fairly insensitive to PMBT, a colder motor results in a lower thrust burn for a longer duration. Conversely, a hotter motor results in a higher thrust for a shorter duration. The hotter motor is the more stressful case for a pad abort, resulting in less downrange because there is less time available to perform the pitch over. An additional dispersion was applied to the burn rate to account for off-nominal performance due to propellant manufacturing variability. This factor affected thrust and burn time but did not change the total impulse.

In addition to AM performance, wind profiles were important in estimating worst-case pad abort performance. For this analysis, a $95^{\text {th }}$ percentile on-shore wind profile was assumed. This wind profile was derived from a set of measured Kennedy Space Center winds and enveloped the on-shore component of $95 \%$ of the low altitude profiles.

Figure 5 shows altitude vs. downrange plots for several pad abort simulations using various combinations of these assumptions. Simulations were terminated at LAS jettison. The case with a nominal AM burn rate and no winds easily meets the requirement. With a high burn rate AM profile (100 deg F PMBT, $+3 \sigma$ burn rate dispersion) and $95^{\text {th }}$ percentile on-shore winds, downrange is reduced by $\sim 500 \mathrm{ft}$. If the initial ACM pitch maneuver is adjusted to pitch faster, enough downrange can be gained to meet the requirement with the worst case burn rate and wind assumptions. The effect of the higher pitch over on the angle-of-attack time history is shown in Fig. 6 . The figure also shows the reorientation and subsequent stabilization at the CM trim angle-of-attack prior to LAS jettison. Future Monte Carlo analyses are planned to ensure that the required pitch maneuver can be performed with adequate control margin to account for dispersions. 


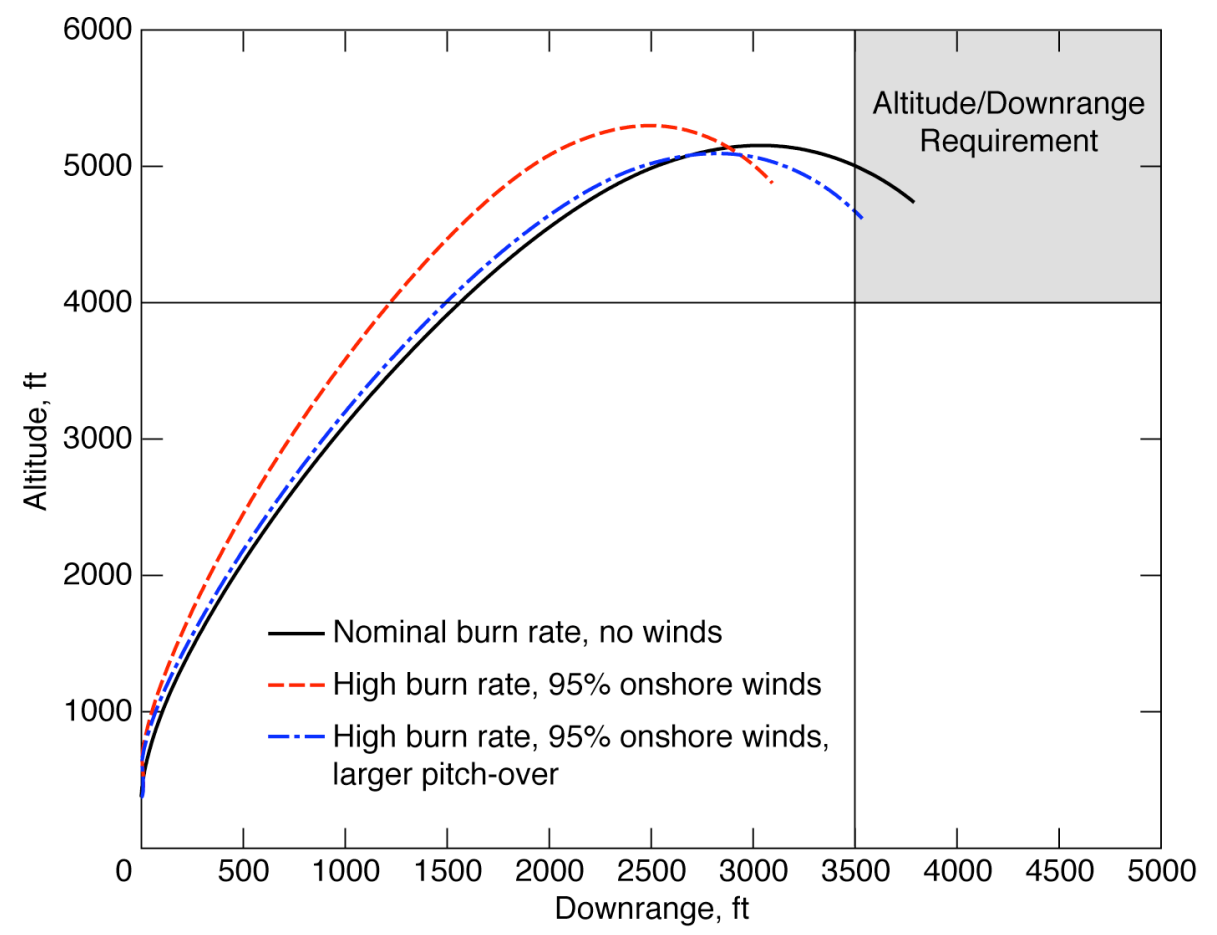

Figure 5. Altitude and downrange performance during a pad abort.

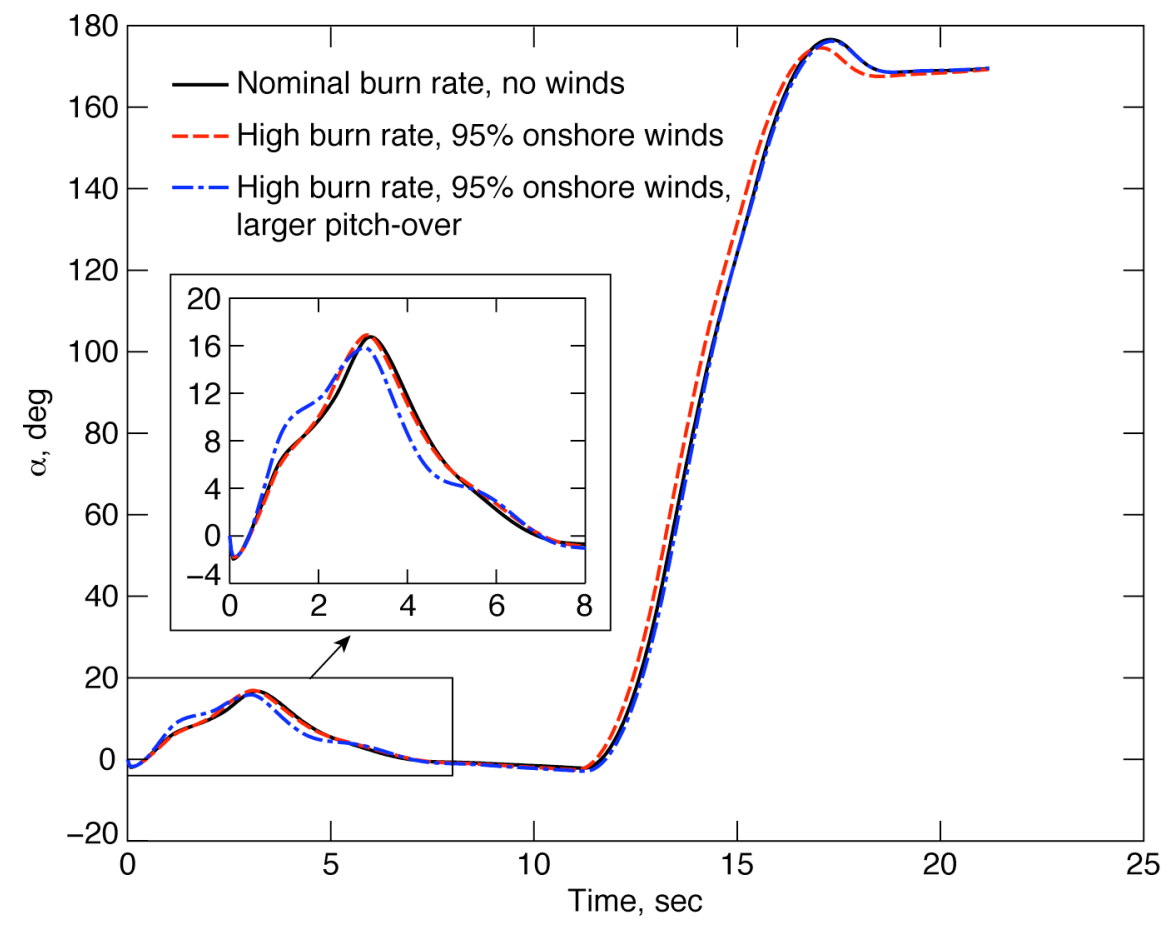

Figure 6. Angle of attack profiles for a pad abort. 


\section{B. Separation Distance Performance}

A key abort performance metric is the ability of the LAV to separate from a thrusting CLV during the high drag levels associated with transonic and high dynamic pressure aborts. In this region of the abort space, the AM must have enough thrust to rapidly overcome drag and accelerate the LAV to an adequate separation distance in order to reduce the chance of re-contact with the failing launch vehicle and minimize exposure to potential debris from a launch vehicle explosion.

As the LAV pulls away from the CLV at abort initiation, the suction induced by the gap between the CM and $\mathrm{SM}$ increases the base drag on the LAV until a sufficient separation distance ( $\sim 50 \mathrm{ft}$, or $3 \mathrm{CM}$ diameters $)$ is achieved. At transonic speeds the proximity drag increment is significant, increasing the overall LAV drag by $40-$ $50 \%$ at abort initiation. The drag coefficient increment $\left(\Delta \mathrm{C}_{\mathrm{D}}\right)$ due to proximity tends to decrease with Mach number; however, when the dynamic pressure profile from the ascent trajectory is factored in, the deceleration due to drag that must be overcome by the LAV peaks at $\sim 7$-g's near Mach 0.9 (see Fig 7).

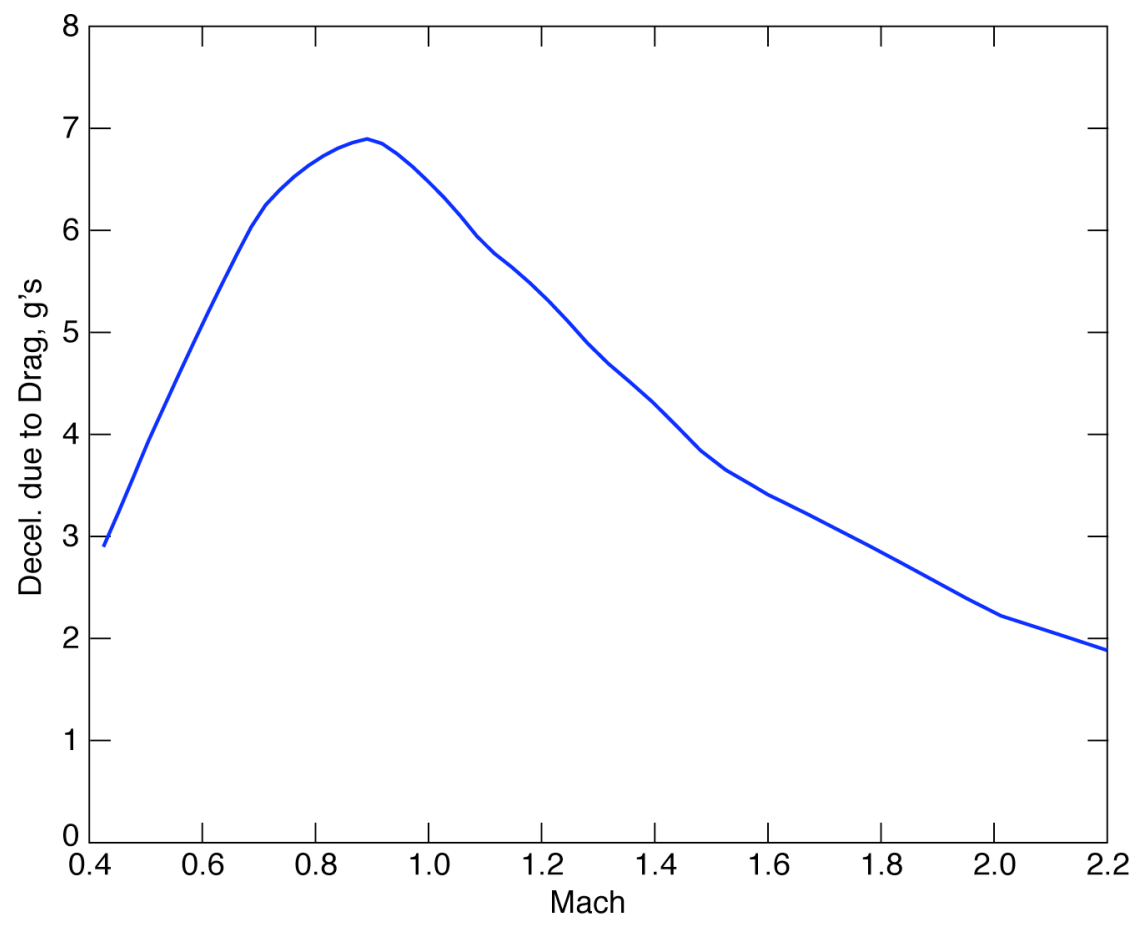

Figure 7. Initial deceleration due to drag that must be overcome in an ascent abort.

Consequently, separation performance for transonic aborts effectively sets the minimum acceptable thrust level of the AM. Some ACM pitch maneuvering can enhance separation performance, but a less aggressive pitch-over must be performed compared to a pad abort since less control margin is available due to the higher dynamic pressure.

The effect of Mach number at abort initiation on separation distance is shown in Fig. 8. These results assumed a

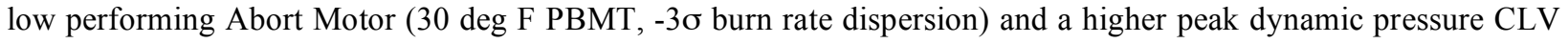
ascent trajectory $(\sim 1000 \mathrm{psf},+3 \sigma)$. The separation distance $3 \mathrm{sec}$ after abort initiation was used as a metric to compare these simulation abort cases. As expected, the worst performance occurs at Mach 0.9 where three seconds after abort initiation the separation distance was $\sim 370 \mathrm{ft}$. For an abort occurring at peak dynamic pressure (near Mach 2.0 in this off-nominal ascent trajectory), the separation distance at $3 \mathrm{sec}$ was higher ( $\sim 625 \mathrm{ft})$ but there was a dip in the separation distance profile to $\sim 550 \mathrm{ft}$ after AM burnout, when the CLV passed the LAV. There was no dip in the separation distance profile for the transonic abort because the CLV passed the LAV sooner, due to the higher proximity drag increment at Mach 0.9 compared to Mach 2.0. 


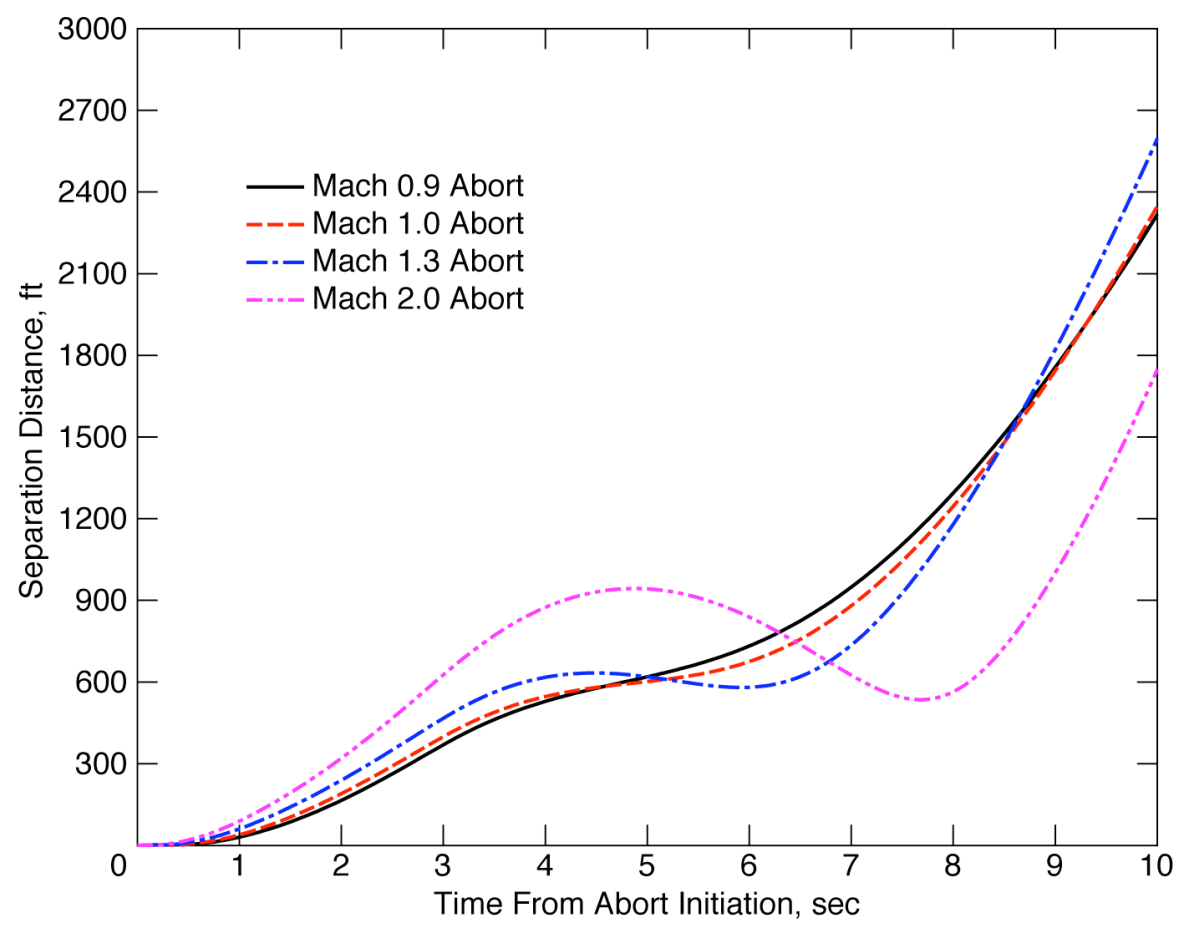

Figure 8. Separation distance performance for a range of ascent abort Mach numbers.

\section{LAS Jettison Performance}

As previously discussed, the LAS Jettison Motor must be designed to achieve acceptable jettison performance during an abort as well as during a nominal mission when an abort is not necessary. The nominal jettison scenario sizes the JM since it must be capable of pulling a fully loaded LAS away from a thrusting CLV. The JM is sized to produce a net acceleration of more than 1.0-g relative to a thrusting CLV (assuming margin for temperature variations and launch vehicle dispersions). The JM thrust vector is offset by 4 deg to provide lateral separation. The offset also induces a rotational rate that causes the LAS to pitch during the jettison burn, further increasing lateral separation.

In these analyses, separation distance was used as the primary performance metric. Figure 9 shows the sensitivity of motor temperature and thrust angle to the separation distance between the LAS and CLV during a nominal jettison maneuver. The separation distance is measured from the CLV, which is accelerating at approximately $0.8 \mathrm{~g}$. The shape of the curves illustrate how the LAS pulls away while the JM is firing and is then passed by the CLV after JM burnout. The results indicate that temperature variations do not have a significant effect on separation performance, largely because the total impulse of the motor doesn't change with temperature. The cold JM thrusts at a lower level for a longer time and thus achieves less axial but more normal separation due to the LAS rotation during the burn. A lower thrust angle reduces the LAS rotation rate during the JM burn and results in $100 \mathrm{ft}$ less lateral separation distance when the CLV passes it.

For jettison during an abort, the LAS separates from the CM after reorientation and ACM burnout, and the JM fires in the opposite direction of LAV motion, acting to slow the LAS relative to the coasting CM. In this scenario, the LAS must also take the docking adapter so that the CM parachutes can deploy. Without the weight of its propellant, jettison of the LAS after an abort results in comparatively better separation performance. Figure 10 illustrates the improved separation distance from the CM in the first five seconds after a pad abort LAS jettison. 


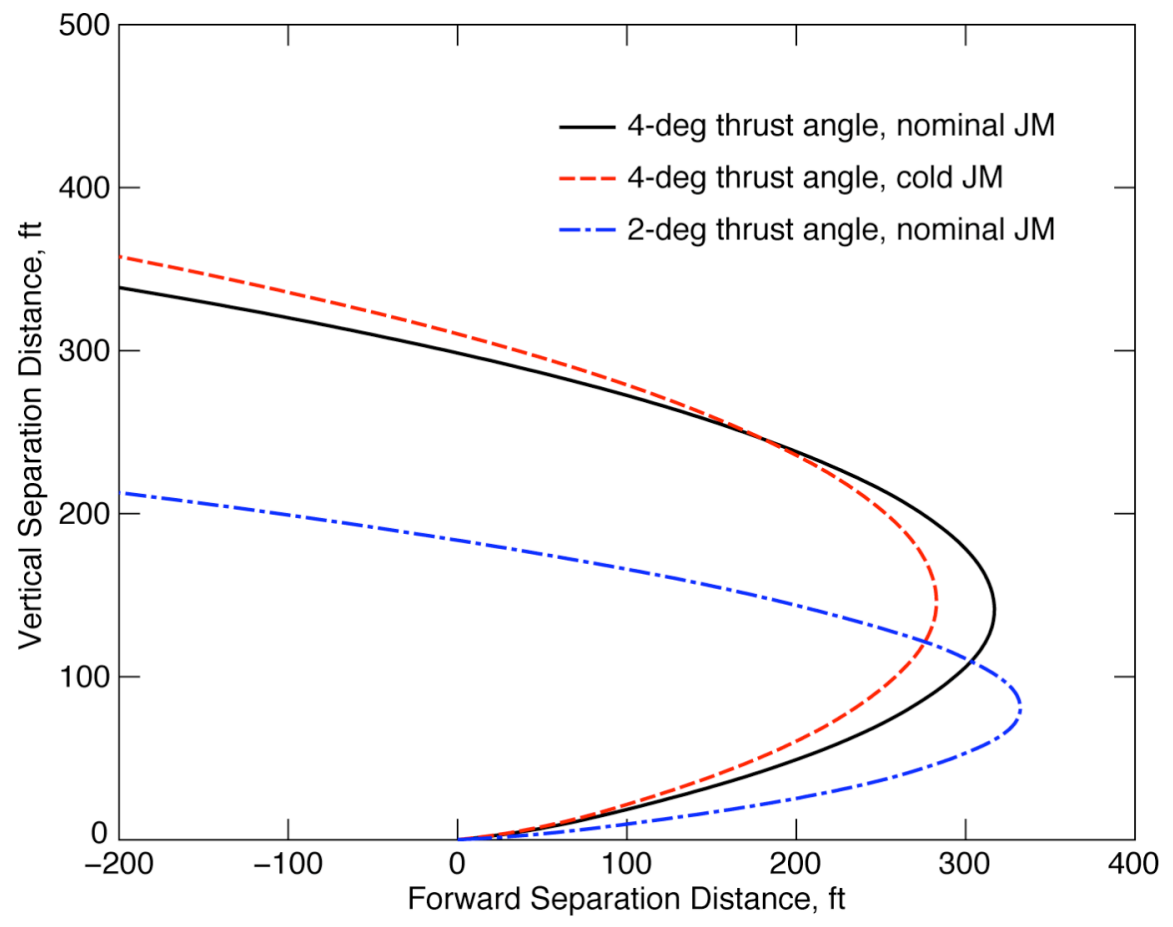

Figure 9. Separation distance performance during a nominal mission LAS jettison event.

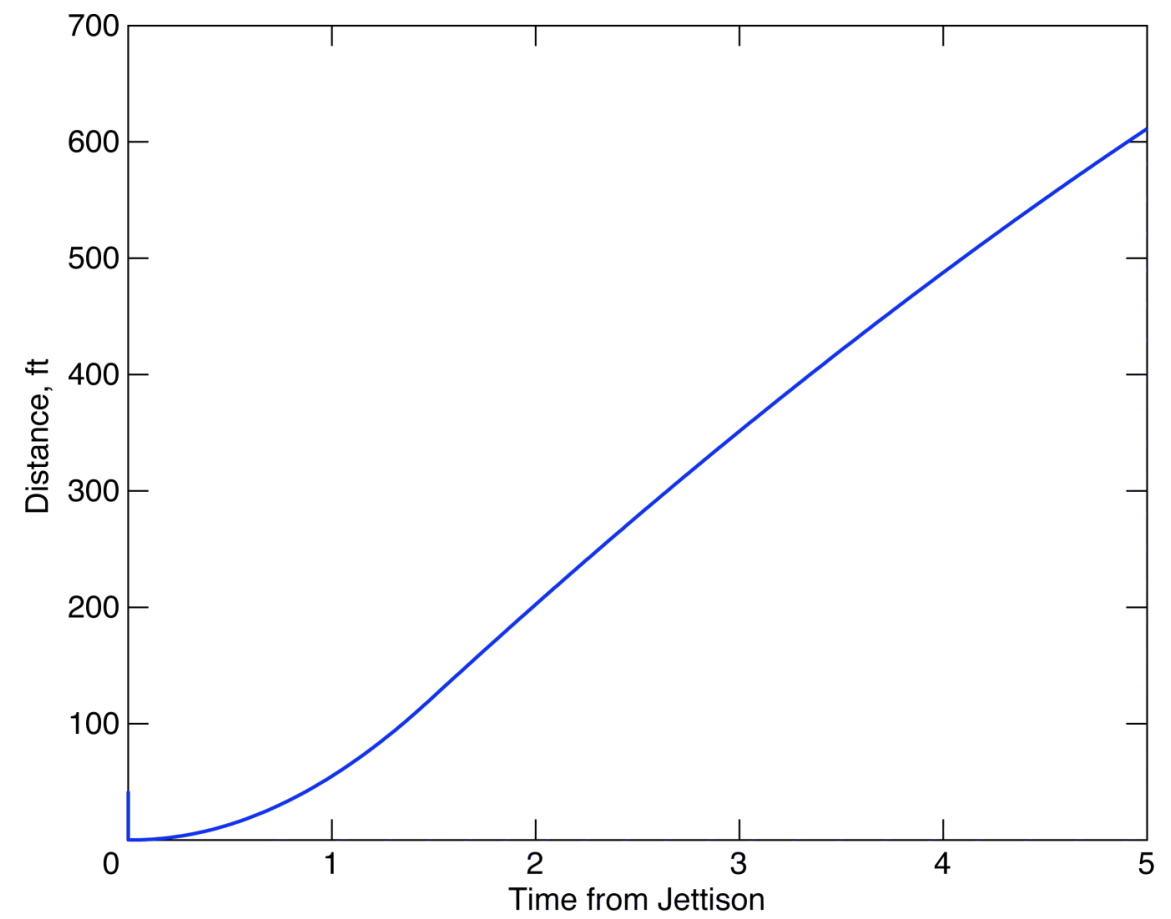

Figure 10. Separation distance performance during a pad abort LAS jettison event. 
In addition to separation performance, the timing of LAS jettison during a nominal ascent is important since it affects launch vehicle performance and abort coverage. To optimize CLV payload capability the LAS weight should be jettisoned as soon as it is no longer needed to ensure a safe crew escape. The earliest that nominal jettison could occur is immediately after first-stage separation, since the high-thrust capability of the AM is needed to assure crew escape from a still-burning SRB during the entire first-stage burn. Of course, it may be prudent to retain the LAS during part of the second-stage burn to further enhance abort capability. The effect of the nominal LAS jettison time on CLV payload performance was estimated by varying the time of jettison and re-optimizing a representative CLV ascent trajectory ( $-30 \times 100 \mathrm{~nm}$ target orbit, 51.6 deg inclination). The resulting payload performance sensitivity is shown in Fig. 11, where the baseline nominal LAS jettison time was assumed to be $30 \mathrm{sec}$ after the upper stage J-2X engine reaches full thrust. The results indicate that payload performance is fairly insensitive for small changes in jettison time, with the delta varying roughly $\pm 150-200 \mathrm{lb}_{\mathrm{m}}$ for cases within $\pm 20 \mathrm{sec}$ of the baseline. However, the performance penalty increases significantly when the LAS is held much longer than $20 \mathrm{sec}$ past the baseline.

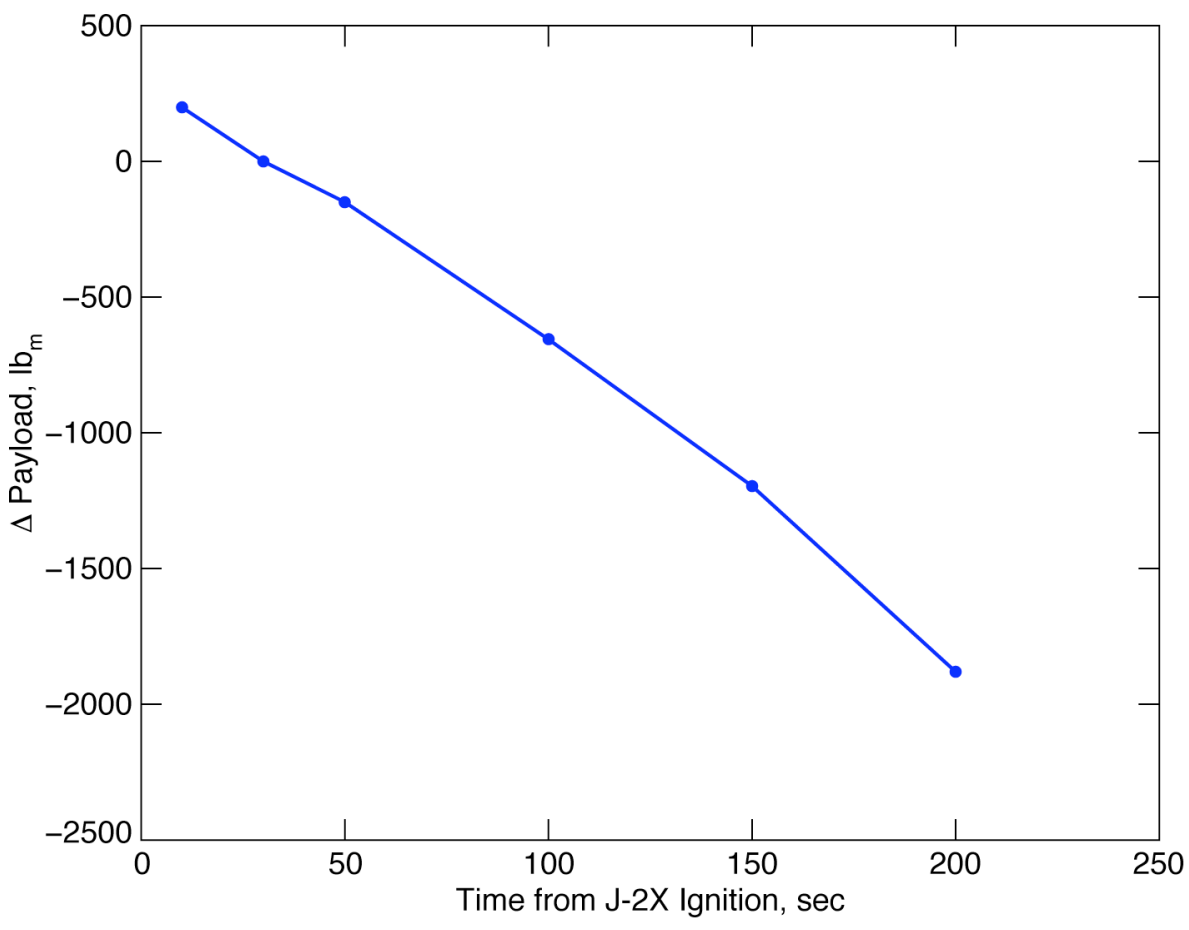

Figure 11. Effect of nominal mission jettison timing on launch vehicle payload performance.

Another consideration with regards to the timing of nominal LAS jettison is the acceleration of the CLV, which tends to build throughout ascent and could potentially degrade separation performance. For jettison times within \pm 20 sec of the baseline, the CLV acceleration varies by only $\pm 5 \%$ and has a minimal effect on separation distance. Another concern is that the LAS should not be released until the SM is capable of performing an abort. For Apollo, abort procedures did not allow for a SM abort until there was at least $100 \mathrm{sec}$ of free fall time (i.e., coast time between abort initiation and atmospheric interface at $300 \mathrm{kft}$ ) to provide ample time for the SM propulsion system to reorient the $\mathrm{CM}$ to its proper atmospheric entry attitude. The actual requirement may be less for Orion since reorientation does not have to be performed manually as in Apollo. The free fall time to $300 \mathrm{kft}$ was computed for the reference ascent trajectory used in this study and found to exceed $100 \mathrm{sec}$ for the range of nominal jettison times that were considered. More detailed SM abort simulations are planned to confirm this result.

These analyses suggest that there may be some freedom in selecting when the LAS should be jettisoned during a nominal trajectory since small deltas $( \pm 20 \mathrm{sec})$ in jettison time do not have a large effect on payload to orbit or the ability to perform a SM abort. It may be possible to delay LAS jettison by 20-30 sec beyond the baseline, thus retaining the option of doing either a LAS abort or a SM abort for roughly the first minute of the upper stage burn. Depending on the failure mode, a SM abort might be preferred to avoid unnecessarily subjecting the crew to high accelerations. 


\section{Conclusion}

This paper provides an overview of the Orion launch abort system and discusses a number of key performance metrics used to assess escape and jettison capability during aborts off the launch pad, at high dynamic pressures and in exoatmospheric conditions.

During a launch pad abort the Abort Motor must have enough total impulse to meet altitude and downrange requirements to ensure successful parachute deployment and landing in safe terrain. Downrange must be achieved by tailoring the abort trajectory with an initial pitch-over maneuver performed using the Attitude Control Motor, a unique feature of the Orion LAS that improves performance robustness compared to the passive approach used in Apollo. Downrange performance was shown to be sensitive to winds and propellant mean bulk temperature, decreasing by $\sim 500 \mathrm{ft}$ with 3- $\sigma$ high temperature and on-shore wind profiles. It was possible to recover the performance through further tailoring of the pitch profile.

The Abort Motor must also produce sufficient thrust early in its burn to avoid collision with the failing launch vehicle and minimize debris exposure during transonic and high dynamic pressure aborts. The drag increase due to separation is highest at transonic speeds where the acceleration margin of the Abort Motor is significantly reduced. Consequently, the Abort Motor thrust profile is designed to have enough thrust at cold conditions to achieve adequate transonic abort separation performance, while at the same time meeting structural design margins and crew safety acceleration limits at hot conditions.

The Jettison Motor must be designed to jettison fully loaded LAS during a nominal mission and a spent LAS during an abort; thus, it is the only LAS motor that will be used on every Orion mission. The Jettison Motor is sized by its nominal jettison function and must provide sufficient thrust and impulse to clear a normally thrusting CLV. The timing of nominal LAS jettison during the ascent trajectory affects launch vehicle performance and abort coverage. Results showed that jettison times ranging from 10 to $50 \mathrm{sec}$ after second stage ignition do not have a substantial impact on payload capability or the ability to perform Service Module aborts after the LAS is jettisoned.

The analyses presented here were performed in support of early concept feasibility studies. The LAS configuration will change as the Orion design is matured, and these issues will continue to be investigated in further detail. Future work will include higher fidelity modeling and account for system dispersions.

\section{Acknowledgments}

The author would like to thank Anne Rhodes for assistance in preparing this paper for publication. The author would also like to acknowledge David Raney and John Davidson for development of the abort control algorithms used in this study, Bruce Jackson and Dana McMinn for assistance in simulation development and Virgil Hutchinson for providing information and clarification on LAS models.

\section{References}

1 “Exploration Systems Architecture Study Final Report,” NASA TM-2005-214062, November 2005.

${ }^{2}$ Davidson, J.B, Madsen, J., Proud, R., Raney, D., Sparks, D., Kenyon, P., Burt, R., Merritt, D. and McFarland, M., “Crew Exploration Vehicle Ascent Abort Overview," AIAA 2007-6590, August 2007.

${ }^{3}$ McCarthy, J. F., Dodds, J. I. and Crowders, R. S., "Development of the Apollo Launch Escape System,” J. Spacecraft, Vol. 5, No. 8, p.927, North American Rockwell Corporation, Downey, CA, Aug. 1968.

${ }^{4}$ Sparks, D.W. and Raney, D. L., “Crew Exploration Vehicle Launch Abort System Controller Performance Analysis,” AIAA 2007-6590, August 2007.

\footnotetext{
${ }^{5}$ Brauer, G. L., Cornick, D. E., and Stevenson, R., "Capabilities and Applications of the Program to Optimize Simulated Trajectories (POST),"NASA CR-2770, Feb. 1977.

${ }^{6}$ Powell, R. W., et. al., "Program to Optimize Simulated Trajectories (POST II) Utilization Manual, Version 1.1.7," July 2002.

${ }^{7}$ McMinn, J.D., Jackson, B. E. and Christhilf, D. M., "Simulation Environment for Orion Launch Abort System Control Design Studies," AIAA 2007-6881, August 2007.

${ }^{8}$ Justus, C. G., Johnson, D. L., The NASA/MSFC Global Reference Atmospheric Model -1999 Version (GRAM-99), NASATM-1999-209630, May 1999.
} 